\title{
Fonction argumentative et fonction figurative de l'analogie : quelle relation entre l'argument par analogie et l'argument par métaphore ?
}

\author{
Philippe Monneret \\ Université Paris-Sorbonne - EA 4509 STIH \\ philippe.monneret@gmail.com
}

\begin{abstract}
Résumé. La question de l'argument par analogie est abordée non pas dans le cadre des théories de l'argumentation mais dans celui d'une théorie de l'analogie. Ce changement de perspective conduit à une clarification de ce type d'argument reposant sur la mise en évidence de deux distinctions cruciales: la distinction entre similarité et analogie d'une part, entre fonction discursive et fonction figurative de l'analogie d'autre part. Ces distinctions permettent de clarifier la relation qui existe entre argument par analogie et argument par métaphore.
\end{abstract}

\begin{abstract}
Argumentative Function and Figurative Function of Analogy: What Relationship between Argument by Analogy and Argument by Metaphor? The question of argument by analogy is discussed not in the framework of the theories of argumentation but in that of a theory of analogy. This change in perspective leads to a clarification of this category of argument based on the demonstration of two crucial distinctions: the distinction between similarity and analogy on the one hand, between discursive function and figurative function of analogy on the other hand. These distinctions make it possible to clarify the relation between argument by analogy and argument by metaphor.
\end{abstract}

L'argument par analogie est une catégorie argumentative traditionnelle qui figure dans toutes les typologies d'arguments, quel que soit le cadre théorique utilisé. En dépit de cette stabilité, la catégorie demeure confusément délimitée, en raison de sa proximité avec d'autres catégories d'arguments qui, selon les auteurs, sont tantôt considérés comme des cas de l'argument par analogie, tantôt distingués de celui-ci. Ainsi, les arguments par l'exemple, par métaphore, par définition et a pari sont parfois distingués de l'argument par analogie, parfois inclus dans cette catégorie - ce qui oblige les auteurs qui choisissent cette dernière solution à opérer une autre distinction, entre une catégorie large de l'argument par analogie et une catégorie de l'argument par analogie au sens restreint incluse dans la précédente, aux côtés de l'argument par métaphore, a pari, etc. Ces variations sont évidemment dues au fait que l'argument par analogie possède une longue histoire et qu'il n'a pas échappé, malgré sa permanence, aux mutations théoriques qui ont affecté la 
rhétorique, la stylistique et les théories de l'argumentation. Nous proposons ici un changement de perspective consistant à examiner l'argument par analogie non pas à la lumière des théories de l'argumentation mais à partir d'une problématisation linguistique de l'analogie, celle-ci étant définie comme un processus cognitif. Ce cadre théorique conduira à accorder une importance de premier plan à la distinction entre analogie et similarité mais aussi à la distinction entre une fonction argumentative et une fonction figurative de l'analogie, cette dernière distinction permettant notamment de construire une différence opératoire entre l'argument par métaphore et l'argument par analogie. Au fond, on a beaucoup écrit sur l'argument par analogie sans avoir pris la peine de construire un concept d'analogie possédant une cohérence suffisante et une certaine plausibilité cognitive. C'est ce genre de lacune que nous tenterons ici de combler.

\section{L'argumentation comme fonction de l'analogie}

Aborder la question de l'argument par analogie à partir d'une « fonction argumentative de l'analogie » suppose en premier lieu que l'analogie soit comprise comme un processus comportant différents types de fonctions. Au sens où elle est documentée par la psychologie cognitive depuis les années 1980 (Gentner 1983), l'analogie possède une fonction psychologique générale consistant à appréhender une situation nouvelle ou mal connue, la cible, en mobilisant des connaissances associées à une autre situation, la source, similaire à la cible. Par exemple, comme l'illustre la figure 1 modélisant l'analogie dite «de Rutherford », certains aspects de la structure de l'atome (notamment le fait que les électrons gravitent autour du noyau) peuvent être compris à partir d'une connaissance préalable de la structure du système solaire. 

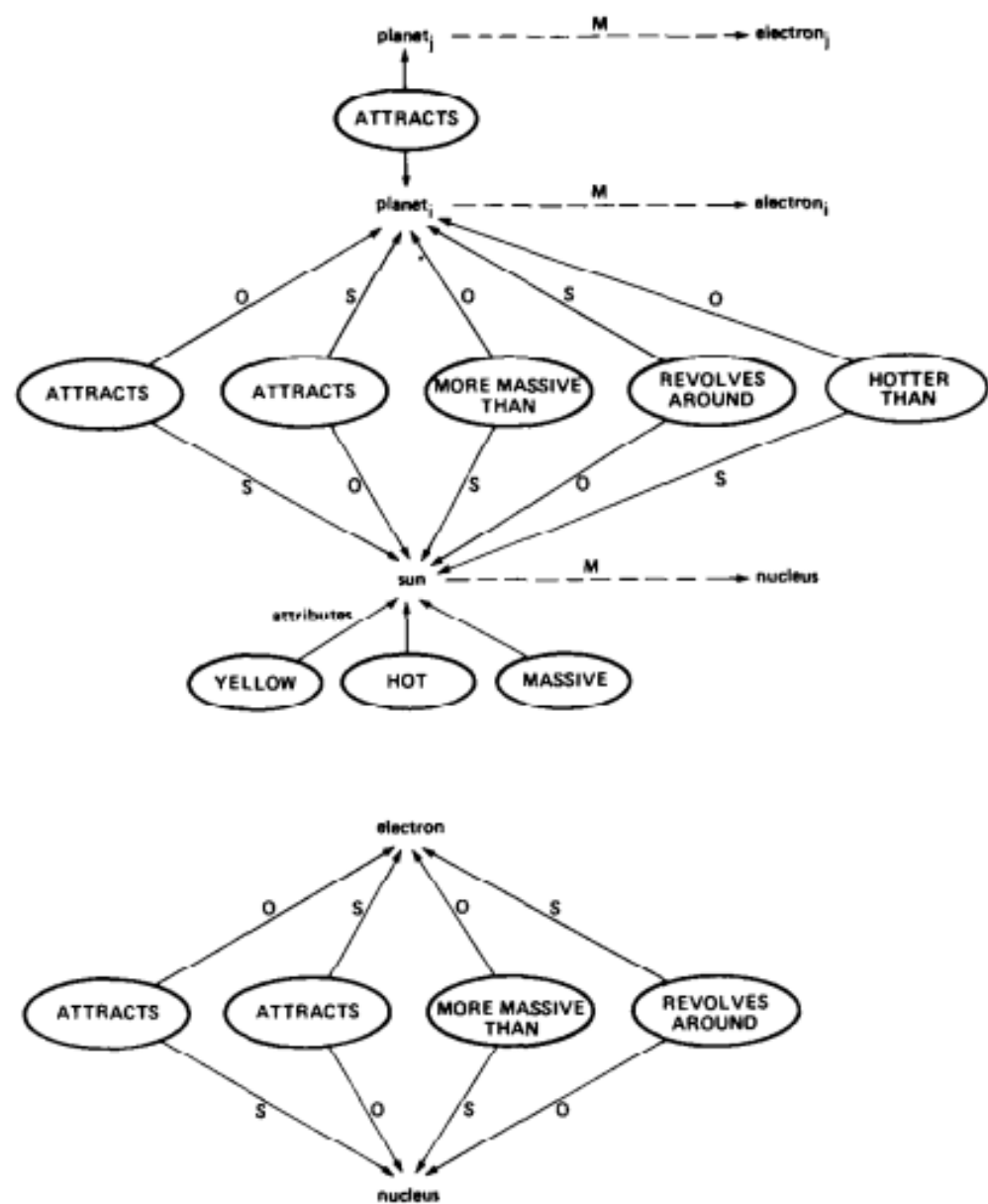

Fig. 1. L'analogie de Rutherford entre l'atome et le système solaire (Gentner 1983, 160)

Cette aptitude aux processus analogiques n'est pas spécifiquement humaine (Truppa et al., 2011) mais elle est particulièrement développée chez l'être humain et joue un rôle central dans la cognition humaine :

The ability to make analogies lies at the heart of human cognition and is a fundamental mechanism that enables humans to engage in complex mental processes such as thinking, categorization, and learning, and, in general, understanding the world and acting effectively on it based on her/his past experience [...] The ability to see a novel experience, object, situation or action as being "the same" as an old one, and then to act in an approximately appropriate manner (and then fine-tuned to fit the novel experience), is, almost unquestionably, one of the capacities that sets humans apart from all other animals. » (Kokinov et French, 2004).

Le processus analogique consiste plus précisément en une mise en correspondance (mapping) de deux structures, comme l'indique la modélisation la plus courante de ce processus (voir fig. 2). Le mapping permet dans certains cas de faire apparaître, par analogie avec la source (donc par inférence), des éléments structurels de la cible non perçus initialement (« candidate inference » dans la fig. 2). 


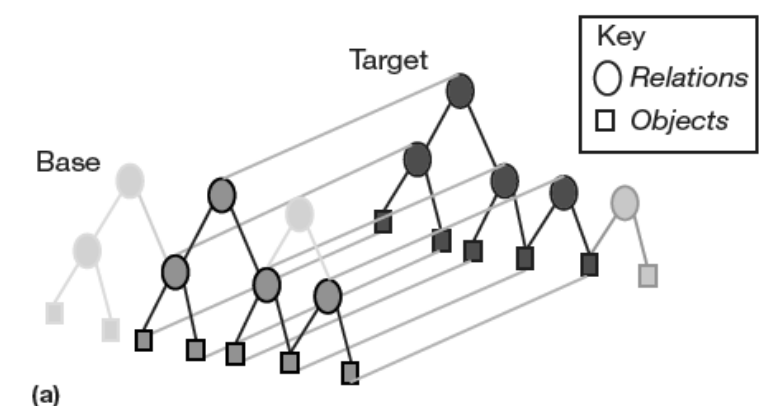

(a)

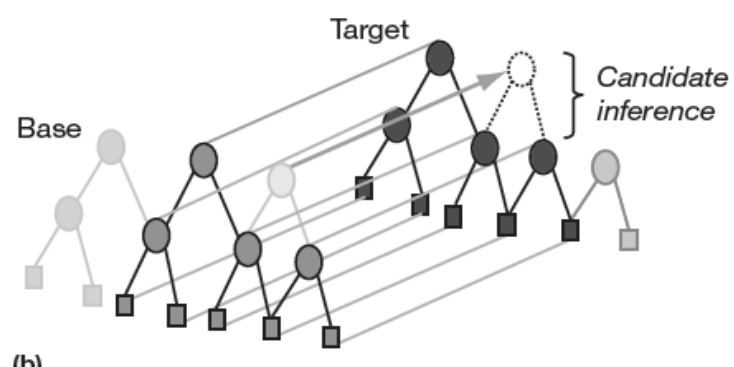

(b)

Fig. 2. Modélisation de l'analogie en psychologie cognitive (Gentner et Smith, 2012)

Les processus analogiques, initialement cantonnés à la description d'un type de raisonnement, ont peu à peu été reconnus comme impliqués dans la catégorisation (Hofstadter et Sander 2013), et par conséquent décrits comme des processus plus souvent non conscients que conscients.

Si l'analogie est un processus central de la cognition humaine («the core of cognition» selon la formule de Hofstadter, 2001), il convient d'en chercher les contreparties linguistiques. Autrement dit, le concept d'analogie au sens cognitif peut être utilisé comme un concept directeur de l'analyse linguistique. Dans une telle perspective, on se demandera donc quels sont les processus et structures linguistiques qui peuvent être rapportés à des processus analogiques. À la fonction psychologique générale de l'analogie, s'adjoignent un certain nombre de fonctions linguistiques plus spécifiques: fonction catégorisatrice, fonction régularisatrice, fonction iconique, fonction paraphrastique, fonction figurative et fonction argumentative (voir Monneret 2004, 2014, 2017a, 2017b).

$\mathrm{La}$ fonction catégorisatrice de l'analogie linguistique est une spécification de la fonction psychologique générale de catégorisation. Elle a fait l'objet d'un examen assez attentif dès les débuts de la linguistique cognitive, avec les travaux de Rosch et de Lakoff principalement. Mais si le rôle de la similarité est explicitement reconnu dans la conception prototypique des catégories (au sens où l'appartenance d'une entité à une catégorie est mesurée par la similarité de cette entité avec un prototype de la catégorie), le rapport fondamental entre analogie et catégorisation semble avoir été largement ignoré. Car la catégorisation, qu'il s'agisse de catégoriser une structure phonologique, morphologique, syntaxique, sémantique, textuelle ou référentielle, n'est rien d'autre qu'un processus analogique. Plus exactement, catégoriser, c'est effectuer une analogie dans un objectif de catégorisation: c'est en ce sens que la catégorisation constitue l'une des fonctions de l'analogie, mais une fonction présentant un caractère fondamental dans la mesure où elle est très souvent sous-jacente aux autres fonctions de l'analogie.

La présentation de cette première fonction nous donne l'occasion de préciser le rapport entre similarité et analogie. La similarité sera définie comme une relation entre deux ou plusieurs entités possédant des propriétés communes, ces propriétés pouvant éventuellement être relationnelles. Par exemple, des similarités peuvent être mises en 
évidence entre la crise économique de 2009 et celle de 1929 (Johsua 2010) ou entre l'abattage des bêtes dans les abattoirs et l'extermination des juifs pendant la seconde guerre mondiale : «Adorno et Horkheimer, Derrida, Canetti, Grossman, Gary, entre autres, ont été obsédés par la douleur animale et par sa proximité avec la souffrance des persécutions par les nazis » (Fontenay 2008). Mais ces similarités sont «neutres »: en elles-mêmes, elles ne plaident ni en faveur de l'identification ni en faveur de la différenciation des situations considérés comme similaires. Dans le cas des crises économique de 2009 et de 1929 , Johsua reconnait qu'il existe des similarités entre ces crises mais qu'elles ne peuvent en aucun cas être confondues, et en particulier qu'on ne saurait se fonder sur l'expérience antérieure de la crise de 1929 pour élaborer une réponse appropriée à la crise de 2009. En revanche, dans le cas de l'abattage des animaux, la plupart des militants antispécistes considèrent, à l'instar de Singer ou de Patterson, que l'expérience des animaux tués dans les abattoirs et celle des juifs tués dans les camps d'extermination sont identiques. Dans une nouvelle d'Isaac Bashevis Singer régulièrement citée par les antispécistes, un personnage s'adresse ainsi à une souris morte : "Tous ces philosophes, les dirigeants de la planète, que savent-ils de quelqu'un comme toi ? Ils se sont persuadés que l'homme, espèce pécheresse entre toutes, domine la création. Toutes les autres créatures n'auraient été créées que pour lui procurer de la nourriture, des fourrures, pour être martyrisées, exterminées. Pour ces créatures, tous les humains sont des nazis ; pour les animaux, c'est un éternel Treblinka » (Singer 2004). Un éternel Trebinka, titre d'un livre de Patterson dont Elisabeth de Fontenay commentait la traduction en français dans l'article de 2008 que nous avons cité. La neutralité de la similarité est donc due au fait que celle-ci implique bien sûr des ressemblances, qui peuvent justifier le rapprochement des situations considérées, mais aussi des différences entre ces situations, différences sans lesquelles la relation entre les situations serait non pas de similarité mais d'identité.

Compte tenu de cette conception de la similarité, l'analogie peut alors être définie comme un processus cognitif d'identification fondé sur des similarités. Ce processus d'identification ${ }^{1}$ est effectué par un sujet, selon un point de vue qui lui est propre et qui peut être plus ou moins largement partagé. Par exemple, dans le cas de l'antispécisme, l'expression "holocauste animal » procède d'un processus analogique fondé sur des similarités objectivables entre les deux situations considérées. Mais on peut fort bien admettre ces similarités sans pour autant accepter l'analogie. Dans le cas de la catégorisation, la similarité entre des situations ou des entités conduira à un processus analogique d'identification catégorielle. La distinction entre similarité et analogie est donc essentielle : elle est constitutive de la définition que nous adoptons de l'analogie. On l'aura remarqué, la proportionnalité n'est pas sollicitée comme critère définitoire de l'analogie au sens où nous l'entendons. L'analogie proportionnelle n'est qu'un cas particulier d'analogie, qui se caractérise par le fait qu'elle est fondée sur des similarités relationnelles (par exemple sur des similarités de relations existant entre des parties d'une situation ou d'une structure et les relations existant entre les parties d'une autre situation ou structure considérée comme similaire) et que l'on peut opposer à l'analogie binaire, fondée sur une similarité issue de propriétés attributives (ou non-relationnelles) communes.

La fonction argumentative sera donc ici considérée comme une fonction de l'analogie au même titre que la fonction catégorisatrice. Outre la fonction figurale de l'analogie, sur laquelle nous reviendrons ultérieurement pour distinguer l'argument par analogie de l'argument par métaphore, les autres fonctions sont, rappelons-le, les suivantes : fonction régularisatrice fonction paraphrastique et fonction iconique. Très succinctement, la fonction régularisatrice prend en charge les régularités des structures linguistiques, principalement morphologiques, aux plans synchronique et diachronique. Elle recouvre en partie la «motivation relative» saussurienne. Lorsqu'une forme nouvelle est produite par un individu (p. ex. ils sontaient produit par un enfant de 18 mois à 3 ans (Leroy-Collombel 
2010, 1548)) ou apparaît dans une langue (p. ex. honor en latin, sur le modèle de formes du type orator, a supplanté l'ancienne forme honos (Saussure 1995, 224)), le processus aboutissant à cette forme nouvelle est un processus analogique fondé sur des similarités existant dans la mémoire du sujet ou dans la langue ou le dialecte considéré (la forme sontaient est attestée en cadien, en québécois, en franco-ontarien, en franco-américain et en franco-minnesotain (Papen et Bigot, 2010)). La fonction paraphrastique prend en charge les processus paraphrastiques intralinguistiques (paraphrase au sens usuel) et interlinguistiques (traduction, voir Ladmiral 2016). Enfin, la fonction iconique concerne les analogies fondées sur des similarités entre formes et contenus ou entre signifiés (ou concepts) et signifiants, et permet en particulier de thématiser la question du symbolisme phonétique, qui fait aujourd'hui l'objet de nombreuses investigations (voir Monneret 2014).

Compte tenu des définitions adoptées, la fonction argumentative de l'analogie consiste en l'exploitation discursive de similarités lexicales, textuelles ou situationnelles. Si la dénomination «fonction argumentative » a été préférée à «fonction discursive », c'est que la dimension argumentative est considérée comme prototypique de l'exploitation discursive de l'analogie. Ainsi, l'usage de l'analogie dans les discours didactiques ou de vulgarisation sont inclus dans ce que nous nommons la «fonction argumentative»de l'analogie.

\section{Traitement de l'analogie dans le cadre des théories rhétoriques et de l'argumentation}

L'une des motivations de cette étude réside dans le fait que les présentations actuellement disponibles de l'argument par analogie présentent des incohérences, que nous mettrons en évidence à partir de quelques auteurs de référence. Les perspectives théoriques générales de ces auteurs seront considérées comme connues. Faute de place, nous nous limiterons au strict examen de la place de l'analogie dans les typologies d'arguments.

Commençons par Perelman et Olbrechts-Tyteca (1958) qui structurent l'ensemble des schèmes argumentatifs à partir d'une opposition entre procédés de liaison et procédés de dissociation. Les premiers, sur lesquels l'analyse est concentrée en raison de leur importance, sont définis de la façon suivante : « Nous entendons par procédés de liaison des schèmes qui rapprochent des éléments distincts et permettent d'établir entre ces derniers une solidarité visant soit à les structurer, soit à les valoriser positivement ou négativement l'un par l'autre » (Perelman et Olbrechts-Tyteca, 1958, 255). Parmi les procédés de liaison, trois types d'arguments sont distingués : les arguments quasi-logiques (incluant notamment l'argument par la définition et l'argument par comparaison), les arguments basés sur la structure du réel et les arguments qui fondent la structure du réel. C'est dans cette dernière rubrique qu'apparaît l'argument par analogie. A la différence des arguments basés sur la structure du réel, qui « se servent de celle-ci pour établir une solidarité entre des jugements admis et d'autres que l'on cherche à promouvoir » (Perelman et Olbrechts-Tyteca, 1958, 351), les arguments qui fondent la structure du réel visent à établir des rapports non préétablis, ou bien en se fondant sur un cas particulier (argumentation par l'exemple ou par l'illustration notamment) ou bien en mobilisant un raisonnement par analogie, qui peut prendre deux formes distinctes: l'analogie ou la métaphore. Le «raisonnement par analogie » est défini traditionnellement à partir de sa structure proportionnelle : « Il nous semble que sa valeur argumentative sera le plus clairement mise en évidence si on envisage l'analogie comme une similitude de structures, dont la formule la plus générale serait : A est à $\mathrm{B}$ ce que $\mathrm{C}$ est à $\mathrm{D}$. Cette conception de l'analogie se rattache à une tradition très ancienne. » (Perelman et Olbrechts-Tyteca, 1958, 500). Quant à la métaphore, elle est présentée comme une sorte de cas limite de l'analogie : « Nous ne pourrions mieux, en ce 
moment, décrire la métaphore qu'en la concevant, tout au moins en ce qui concerne l'argumentation, comme une analogie condensée, résultant de la fusion d'un élément du phore avec un élément du thème » (Perelman et Olbrechts-Tyteca, 1958, 535). Au total, Perelman et Olbrechts-Tyteca incluent donc, dans la rubrique de l'analogie, un argument par analogie proprement dit et l'argument par métaphore ; ils excluent par ailleurs de cette rubrique l'argument par la définition et l'argument par comparaison.

Dans le cas de Toulmin, nous nous appuierons non pas sur The uses of arguments (Toulmin 1958), qui ne contient pas de typologie des arguments explicite mais sur $A n$ introduction to reasoning (Toulmin, Rieke, Janik 1984). Dans cet ouvrage, une classification des arguments est proposée comportant cinq rubriques majeures et quatre rubriques complémentaires («other possible classifications»). Les cinq formes de raisonnement principales sont les suivantes : "Reasoning from analogy », "Reasoning from generalization », "Reasoning from sign» (par exemple adopter un comportement approprié en voiture en interprétant les panneaux de la circulation), «Reasoning from cause », «Reasoning from authority». Les formes complémentaires sont le dilemme, l'argument fondé sur une classification, l'argument tiré des contraires, l'argument selon le degré.

A la différence de Perelman, l'analogie n'est pas définie comme structure proportionnelle mais à partir de la notion de similarité : «In "arguing from analogy," we assume that there are enough similarities between two things to support the claim that what is true of one is also true of the other." (Toulmin et al. 1984, 216). Cette conception de l'analogie semble donc du même ordre que celle que nous avons adoptée : l'analogie est un processus fondé sur des similarités à partir desquelles une identification est opérée, l'identification étant ici relative à la vérité des situations similaires. Cependant, dans le chapitre qu'ils consacrent aux « fallacies », et en particulier à la « false analogy », Toulmin et al. (1984) incluent les analogies dans la catégorie des comparaisons : " Analogies are comparisons that enrich our language and have the power to enlighten our understandingwhen they are appropriate and successful. Sometimes they work on us through similes or metaphors. Think of likening the lion to a king in the phrase "king of beasts," or the state to a ship in the phrase "the ship of state," or the German World War I I commander Field Marshal Erwin Rommel to a fox in the nickname "The Desert Fox" » (Toulmin et al. 1984, 161). La relation logique que nous avons établie entre similarité et analogie est donc ici perdue de vue puisque l'analogie est mise sur le même plan que la comparaison, que nous interprétons dans ce contexte comme un équivalent de la similarité.

Dans cette typologie, l'argument par analogie est une catégorie simple, qui n'inclut aucune autre sous-catégorie d'argument. On pourrait à la rigueur considérer, sur la base de certaines illustrations ${ }^{2}$, qu'elle inclut implicitement l'argument par l'exemple, mais cet argument ne figure pas comme tel dans la typologie. L'argument par comparaison non plus, bien que, comme nous l'avons vu, l'analogie soit elle-même considérée comme une structure comparative. De même, les arguments par la définition et par métaphore sont absents de la typologie, mais la dimension métaphorique apparaît - nous reviendrons sur ce point - comme un aspect facultatif de l'argument par analogie.

Philippe Breton (2009) présente graphiquement sa typologie d'arguments (fig. 3) qui comporte quatre grandes «familles d'arguments»: les arguments d'autorité, de communauté, de cadrage et d'analogie. Cette dernière catégorie est définie de la façon suivante : "L'argument d'analogie consiste à établir entre deux zones du réel jusque-là disjointes une correspondance qui va permettre de transférer à l'une les qualités reconnues à l'autre » (Breton 2009, 95). On reconnait ici l'influence de Perelman et Olbrechts-Tyteca, qui conçoivent l'argument par analogie comme un argument qui «fonde la structure du réel ». Mais, à la différence de ces auteurs, Breton ne caractérise pas l'analogie par une 
structure proportionnelle : «nous employons le terme « analogique » ici au sens le plus large. Il sert à désigner le fait que l'opinion que l'on veut défendre peut être mise « en rapport » avec une opinion, ou une réalité, qui, elle, est admise par l'auditoire » (Breton 2009, 97).

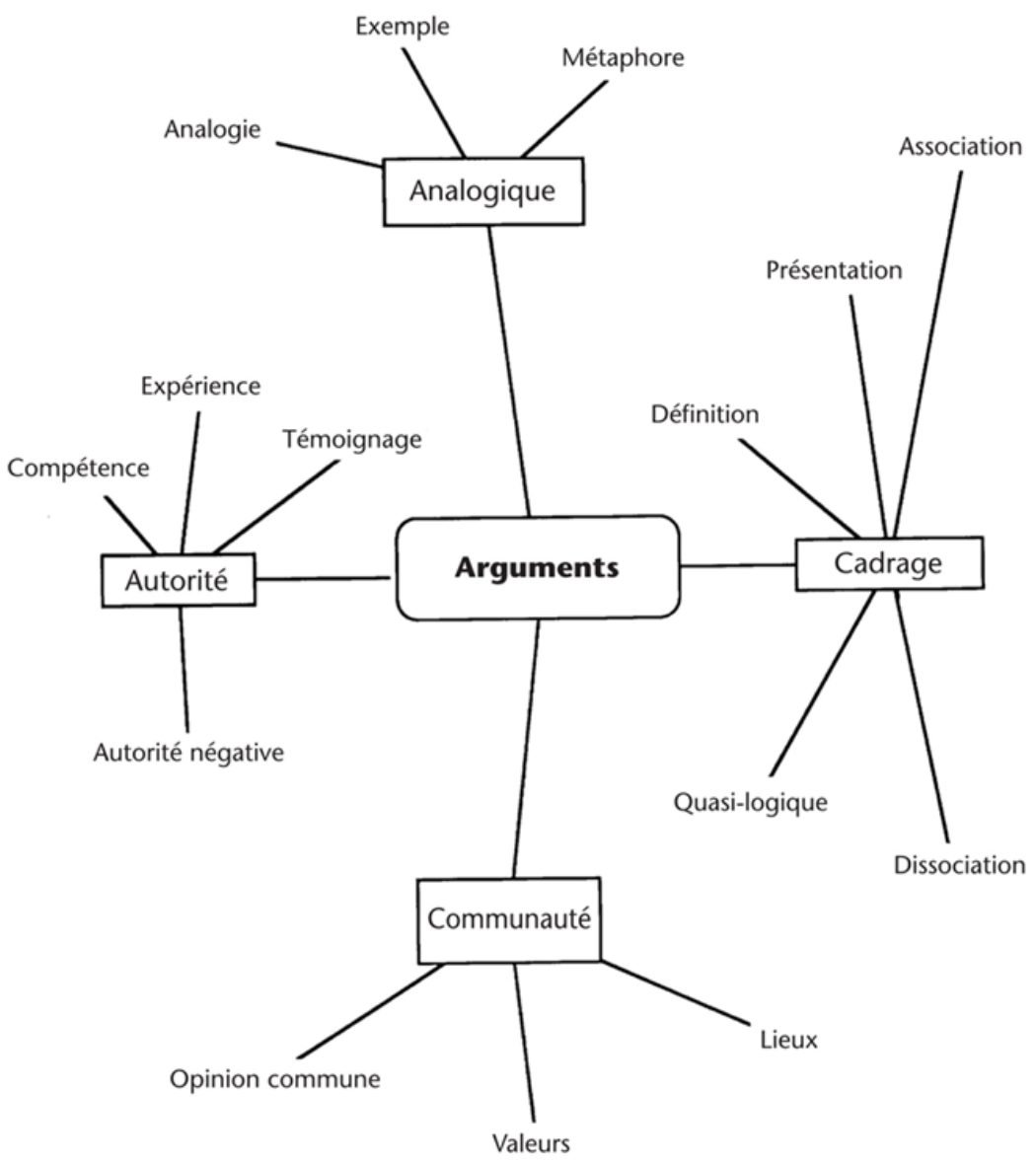

Fig. 3. Les grandes familles d'arguments selon Breton $(2009,45)$

Comme on peut le constater sur la figure 3, l'argument par analogie inclut trois cas distincts: l'analogie proprement dite, l'exemple et la métaphore. L'argument par comparaison ne figure pas dans la typologie. L'argument par définition se situe hors de la catégorie de l'argument par analogie puisqu'il est rattaché à la famille des arguments de cadrage. Compte tenu de la définition adoptée de l'argument par analogie (au sens large), il est naturel que l'exemple en représente un cas puisque ce type d'argument est fondé sur le rapport établi entre un ou plusieurs cas particuliers et un ensemble de cas (en d'autres termes, il s'agit d'une généralisation) :

Ainsi, si je soutiens que « tout le monde, en Amérique, peut faire fortune », je peux, pour convaincre, utiliser l'argument par l'exemple qui énoncerait ici que Bill Gates (l'une des plus grandes fortunes américaines) ou Mark Zuckerberg, qui a créé Facebook (et qui est l'un des plus jeunes milliardaires du monde), « sont des jeunes gens partis de rien et qui sont maintenant parmi les hommes les plus riches du monde ». 
Bill Gates et Mark Zuckerberg représentent ici des exemples potentiellement généralisables (ce qui ne signifie pas que tout le monde deviendra milliardaire, mais que tout le monde peut le devenir, ce qui n'est pas la même chose). Dans un premier temps, on rappelle un fait connu (ces deux hommes sont des milliardaires) et on le place en « posture de généralisation ». Le fait est qu'ils sont milliardaires, l'argument consiste à énoncer que ce qu'ils ont fait est faisable par d'autres. Derrière Gates et Zuckerberg, en filigrane, il y a tout Américain potentiellement milliardaire. L'argument par l'exemple met donc en œuvre une analogie (au sens large) entre «tout le monde en Amérique » et les personnes particulières Gates et Zuckerberg, promues au rang d'exemples. (Breton 2009, 96)

Quant à la métaphore, elle est comprise comme une « ellipse d'analogie » (Breton 2009, 98), ce que vise à illustrer l'exemple suivant: "Ainsi, dire de quelqu'un « quel âne ! " suppose une analogie entre le comportement buté de l'âne (ce que tout le monde peut constater) et celui de telle personne, que ne caractériserait pas son intelligence ou son ouverture » (ibid.). Comme le suggère le commentaire de cet exemple, dire de quelqu'un «quel âne!» revient à effectuer un type particulier d'analogie, que Breton nomme «métaphore » en raison du fait que le rapport entre la source («âne ») et la cible (la personne visée) n'est pas explicité.

Examinons pour finir une dernière approche, issue du tout récent Dictionnaire de l'argumentation de Christian Plantin (Plantin 2016). Ce dictionnaire comporte une entrée « analogie », qui reprend assez largement Plantin (2011) et se subdivise en quatre parties : la première est consacrée à la "pensée analogique », dans un sens proche de celui que lui donne Foucault (1966) dans le cadre des similitudes qui caractérisent l'épistémè du XVI siècle, la deuxième à une description formelle des analogies observables en discours et aux dénominations utilisées pour désigner les relations analogiques, la troisième à l'analogie « catégorielle» et la quatrième à l'analogie «structurelle ». L'analogie catégorielle est définie comme " une relation qui lie les individus appartenant à une même catégorie. Elle est à la base de l'application de l'argument a pari » (Plantin 2016, 52). Quant à l'analogie structurelle, Plantin la conçoit comme la mise en relation de deux domaines complexes « articulant chacun un nombre indéfini et illimité d'objets et de relations entre ces objets » (Plantin 2016, 55). Dans un passage particulièrement intéressant pour notre propos, Plantin $(2016,51)$ énumère plusieurs « formes argumentatives exploitant l'analogie » : i) l'analogie catégorielle, à laquelle il associe l'argumentation par l'exemple; ii) l'analogie de proportion; iii) l'analogie structurelle à laquelle il associe la métaphore. Compte tenu du fait qu'il considère que l'analogie catégorielle est impliquée dans l'argument a pari, et que, par ailleurs, l'argument par la définition se distingue très difficilement de l'analogie catégorielle ${ }^{3}$, nous pouvons distinguer, dans la catégorie générale des arguments par analogie, six types différents dont certains entretiennent d'étroites relations avec d'autres : 1) l'analogie catégorielle ; 2) l'argumentation a pari (lié à l'analogie catégorielle); 3 ) l'argumentation par l'exemple (liée également à l'analogie catégorielle); 4) l'analogie de proportion; 5) l'analogie structurelle; 6) la métaphore (considérée comme un cas particulier de l'analogie structurelle).

Le bilan de cette analyse est présenté dans le tableau 1 ci-dessous. Dans ce tableau, le signe / $+/$ indique que le cas est explicitement présent dans la typologie considérée, le signe /-/ qu'il est absent et le signe $/ \varnothing /$ qu'il n'est pas pertinent. Le signe est mis entre parenthèses si la présence ou l'absence de la rubrique n'est pas explicite. La colonne « analogie » renvoie aux cas où une rubrique spécifique « analogie » est incluse dans une catégorie plus large « argument par analogie ».

Tableau 1. Traitement de l'argument par analogie chez quelques auteurs de référence. 


\begin{tabular}{|l|c|c|c|c|c|}
\hline $\begin{array}{l}\text { Cas inclus dans la } \\
\text { catégorie « argument } \\
\text { par analogie » }\end{array}$ & $\begin{array}{l}\text { Arg. par } \\
\text { l'exemple }\end{array}$ & $\begin{array}{l}\text { Arg. par } \\
\text { métaphore }\end{array}$ & $\begin{array}{l}\text { Arg. par la } \\
\text { définition }\end{array}$ & $\begin{array}{l}\text { Arg. par } \\
\text { comparaison }(a \\
\text { pari })\end{array}$ & Analogie \\
\hline $\begin{array}{l}\text { Perelman et } \\
\text { Olbrechts-Tyteca } \\
(1958)\end{array}$ & - & + & - & - & + \\
\hline Toulmin et al (1984) & $(+)$ & $(+)$ & $\emptyset$ & $(+)$ & - \\
\hline Breton (2009) & + & + & - & $(+)$ & + \\
\hline Plantin (2016) & + & + & + & + & + \\
\hline
\end{tabular}

\section{Fonction argumentative et fonction figurative de l'analogie : le cas de l'argument par métaphore}

Ce tableau récapitulatif permet de prendre la mesure des variantes que connaît, selon les auteurs considérés, l'analyse de l'argument par analogie. Et encore, nous nous sommes limités à quelques références postérieures aux années 1950 : les divergences s'accroitraient sans doute si l'on adoptait un empan historique plus large. Il se dégage globalement une impression de confusion, reposant essentiellement sur l'instabilité de la définition de l'analogie. Nous ne pouvons commenter ici toutes les variations qui apparaissent sur ce tableau : on se limitera à l'examen du contraste entre argument par analogie et argument par métaphore.

La plupart des auteurs, à l'exception de Toulmin, analysent l'argument par métaphore comme un cas particulier d'argument par analogie ${ }^{4}$. Pour Perelman et Olbrechts-Tyteca $(1958,535)$, la métaphore est « une analogie condensée, résultant de la fusion d'un élément du phore avec un élément du thème ». Toulmin et al. (1984) ne distinguent pas un « argument par métaphore » comme cas particulier de l'argument par analogie : ce type d'argument est absent de leur typologie. La métaphore est rapidement mentionnée dans un chapitre consacré aux «stratégies linguistiques » (Toulmin et al. 1984, 206-207) et la seule remarque éclairante pour notre propos est la suivante, formulée au sujet des analogies et des comparaisons : " Sometimes they work on us through similes or metaphors » (Toulmin et al.1984, 161). La métaphore apparaît donc comme une forme linguistique de surface s'ajoutant facultativement à un argument par analogie. Breton $(2009,98)$ quant à lui, la considère comme une «ellipse d'analogie », et Plantin propose la solution suivante, assez proche de celle de Perelman et Olbrechts-Tyteca :

Comme l'analogie, la métaphore argumentative transfère le langage du domaine Ressource (métaphorique) vers le domaine Problème (métaphorisé), mais alors que l'analogie maintient séparés les deux domaines, cette métaphore pousse à les identifier (Plantin 2016, 385).

La force argumentative de la métaphore tient non seulement à ce que, comme l'analogie structurelle, elle introduit un modèle de la situation ciblée, mais en ce qu'elle pousse l'analogie jusqu'à l'identification. C'est en cela qu'elle produit un effet de restructuration du réel ; les êtres mutent de catégories, et en recatégorisant 
radicalement les objets, la métaphore permet de leur appliquer tous les traits de la nouvelle catégorie. [...] L'analogie est une invitation à observer le Problème à travers la lunette de la Ressource : la métaphorisation permet d'oublier la lunette. ( $i$ Plantin 2016, 387)

L'idée d'une fusion ou d'une identification de la source et de la cible de l'analogie peut-elle être utilisée comme un critère distinctif de l'argumentation par métaphore ? Dans notre perspective, compte tenu de la définition de l'analogie que nous avons adoptée, la réponse est clairement négative puisque la tendance à l'identification est définitoire de toute analogie. D'ailleurs, Perelman et Olbrechts-Tyteca $(1958,540)$ semblent parfois proches de cette position :

Toute analogie - hors celles qui se présentent dans des formes rigides, telles l'allégorie, la parabole - devient spontanément métaphore. C'est même l'absence de fusion qui nous obligerait à voir dans l'allégorie, dans la parabole, des formes conventionnelles où la fusion est, par tradition, systématiquement refusée. Loin que l'allégorie soit une métaphore, nous aurions en elle une double chaîne se déroulant avec un minimum de contacts. Il y a dans l'analogie, par son prolongement même, une action qui tend à la fusion.

La solution que nous proposons consiste à faire intervenir une autre fonction de l'analogie : la fonction figurative. Cette solution vise à décrire l'argument par métaphore comme un processus dans lequel interviennent simultanément deux fonctions de l'analogie: la fonction argumentative et la fonction figurative. Ces deux fonctions peuvent évidemment être mobilisées séparément : comme tous les auteurs cités ici le soulignent, une métaphore ne constitue pas nécessairement un argument (dans ce cas, seule la fonction figurative est activée) et, inversement, un argument par analogie n'est pas nécessairement métaphorique (dans ce cas la fonction argumentative de l'analogie est mobilisée mais pas la fonction figurative). Bien entendu, on aura compris qu'une analogie linguistique peut être utilisée sans fonction argumentative : tel est le cas lorsque seule la fonction figurative est activée, mais la remarque vaut également pour les autres fonctions linguistiques de l'analogie.

$\mathrm{La}$ fonction figurative de l'analogie est une fonction visant à produire chez l'allocutaire un effet d'image. Nous ne pouvons ici détailler les rapports complexes mais fondamentaux qui existent, au plan cognitif, entre le concept d'image et le concept d'analogie. On admettra aisément, en première approximation, que l'image implique une similarité au sens où une image d'un objet entretient une relation de similarité avec cet objet. Il est d'ailleurs aujourd'hui habituel, en psychologie cognitive, que la perception et la mémorisation des images visuelles soient considérées comme relevant d'un codage analogique (voir p. ex. Launay 2004, 52). Le cas des images verbales est évidemment distinct de celui des images visuelles puisque l'image verbale, au sens où nous l'entendons ici, est une configuration langagière produisant un effet d'image. Il s'agit donc d'un effet d'image produit par des stimuli fondamentalement sonores, ce qui signifie que l'image est conçue indépendamment de la nature des stimuli, qui peuvent fort bien ne pas être de type visuel. Certaines unités lexicales suscitent spontanément une image mentale lorsqu'elles présentent un degré élevé d'imageabilité ${ }^{5}$ (Bonin et al., 2003 ; Bonin, 2016) : pomme présente un degré élevé d'imageabilité à la différence de anatomie par exemple. On notera en outre que l'imageabilité ne se confond pas avec la concrétude : ange est une unité lexicale perçue comme ayant une bonne imageabilité mais une faible concrétude tandis que l'unité lexicale bombe est inversement perçue comme dotée d'une faible imageabilité mais d'une concrétude élevée. Indépendamment d'autres facteurs que nous ne pouvons pas aborder ici [des références bibliographiques de l'auteur seront ultérieurement ajoutées], la métaphore produit un effet d'image parce qu'elle résulte d'une analogie fondée sur une 
similarité avec une source présentant un degré d'imageabilité plus élevé que la cible (mais aussi plus élevée qu'une dénomination plus « orthonymique » (Pottier 1987, 45). En outre, comme dans toute analogie, la source choisie permet de projeter certaines de ses propriétés sur la cible. Considérons par exemple, la métaphorisation de la société à partir de la cible « corps humain $»^{6}$ :

«Le peuple s'était séparé des sénateurs, pour s'affranchir des impôts et du service militaire, et l'on tentait, pour le rappeler, d'inutiles efforts. " Un jour, dit Agrippa député vers lui, les membres du corps humain, voyant que l'estomac restait oisif, séparèrent leur cause de la sienne, et lui refusèrent leur office. Mais cette conspiration les fit bientôt tomber eux-mêmes en langueur; ils comprirent alors que l'estomac distribuait à chacun d'eux la nourriture qu'il avait reçue, et rentrèrent en grâce avec lui. Ainsi le sénat et le peuple, qui sont comme un seul corps, périssent par la désunion, et vivent pleins de force par la concorde.» Cet apologue ramena le peuple, qui cependant créa des tribuns de son ordre pour défendre sa liberté contre l'orgueil des nobles. » (Sextus Aurelius Victor, cité par Plantin $(2016,387)$ ).

Cette métaphore présente l'intérêt non seulement de susciter une image en raison de l'accroissement d'imageabilité de " société », la cible, à " corps humain », la source, mais aussi de projeter, en raison de la fonction catégorisatrice de l'analogie, certaines propriétés caractérisant le corps humain sur la société : dans cet exemple, le contenu de la projection est l'idée que la coordination entre l'ensemble des membres du corps est nécessaire au bon fonctionnement de l'organisme. Cette métaphore, qui pourrait fort bien être utilisée dans un texte dénué de toute dimension argumentative, est ici utilisée comme argument en faveur de l'idée de coopération entre le peuple et les sénateurs. Par conséquent, elle sollicite également la fonction argumentative de l'analogie.

Le point de vue cognitif que nous avons adopté semble donc permettre de clarifier certains aspects de la problématisation de l'argumentation par analogie. Nous avons en premier lieu distingué similarité et analogie : l'analogie est un processus d'identification fondé sur des similarités. Dépourvu de la distinction de ces deux plans - le plan « descriptif» de la similarité et le plan « processuel » de l'analogie - le concept d'analogie nous semble trop pauvrement défini pour être opératoire. Cette distinction entre similarité et processus analogique d'identification ne peut donc être utilisée pour distinguer l'argument par analogie de l'argument par métaphore. Par ailleurs, nous avons décrit plusieurs fonctions de l'analogie, dont trois sont pertinentes pour la question examinée ici : la fonction catégorisatrice, la fonction argumentative et la fonction figurative de l'analogie. Dans certaines situations langagières, chacune de ces fonctions peut être mobilisée séparément. Mais dans d'autres situations, ces fonctions sont mobilisées simultanément. Le fait qu'une analogie soit mobilisée dans une situation présentant un degré minimal d'argumentativité sera donc décrit comme la mobilisation de la fonction argumentative de l'analogie. Mais comme la plupart des processus analogiques ont des effets de catégorisation (au sens où ils sont perçus comme des catégorisations), l'argument par analogie mobilise également la fonction catégorisatrice de l'analogie : la source et la cible, étant liées par des relations de similarités, sont alors perçues comme membres d'une même catégorie, ce qui autorise l'inférence de certaines propriétés de la source à la cible. Ce type d'identification constitue d'ailleurs le trait caractéristique de la fonction catégorisatrice de l'analogie. Certains des travaux que nous avons mentionnés sollicitent bien le concept de catégorisation, notamment ceux de Plantin, mais la hiérarchisation des concepts d'analogie et de catégorisation demeure confuse : la notion d'analogie "catégorielle » proposée par Plantin (2016, 53-54) est impossible à distinguer d'un argument par analogie qui ne serait pas «catégoriel» puisque, dans la sphère argumentative, toute analogie implique une 
catégorisation potentielle. Enfin, la description en termes de «fonctions de l'analogie» permet de caractériser plus précisément l'argument par métaphore: il s'agit tout simplement d'un argument par analogie doté en outre d'une fonction figurative. La teneur figurative de l'analogie, liée notamment au degré d'imageabilité de la source, nous semble donc gagner à être distinguée des inférences liées à la fonction catégorisatrice de l'analogie.

En termes de typologie d'arguments, nous sommes donc conduits à préconiser une rubrique «argument par analogie», correspondant à la fonction argumentative de l'analogie (rubrique incluant notamment le cas particulier de l'argument par l'exemple ou «par le précédent ») et une rubrique « argument par métaphore », correspondant au cumul de la fonction argumentative et de la fonction figurative de l'analogie. On recommandera en outre l'élimination des rubriques du type "argumentation par catégorisation", « argumentation par la définition», "argument par la comparaison », que notre analyse conduit à considérer comme des formulations surnuméraires de l'idée de catégorisation, qui est impliquée, comme « fonction catégorisatrice », dans toute argumentation par analogie.

\section{Références bibliographiques}

Bonhomme, M., Paillet, A.-M., Wahl, Ph. (éds.). (2017). Métaphore et argumentation, Louvain-laNeuve : Academia, coll. «Au cœur des textes ».

Bonin P., Méot A., Aubert L.-F.., Malardier N., Niedenthal P. M., Capelle-Toczek M.-C. (2003). Normes de concrétude, de valeur d'imagerie, de fréquence subjective et de valence émotionnelle pour 866 mots. L'année psychologique, vol. 103, n4, 655-694.

Bonin, P. (2016) De l'utilité des normes psycholinguistiques pour l'étude de l'accès au lexique mental. AL-Lisaniyyat, vol. 22, $\mathrm{n}^{\circ} 22,59-78$.

Fontenay, E. de (2008) Charles Patterson : l'abattage, un laboratoire de la barbarie, Le Monde des livres, 10 janvier 2008, http://www.lemonde.fr/livres/article/2008/01/10/charles-patterson-labattage-un-laboratoire-de-la-barbarie 997729 3260.html [consulté le 23 mars 2018]

Foucault, M. (1966) Les mots et les choses. Paris : Gallimard.

Gentner, D. (1983) Structure-mapping: a theoretical framework for analogy. Cognitive Science 7, 155-170.

Gentner, D., Holyoak, K.J., Kokinov, B.N., 2001. The analogical mind: perspectives from cognitive science. Cambridge : MIT Press,.

Gentner, D. et Smith, L. (2012). Analogical reasoning. In V. S. Ramachandran (éd.) Encyclopedia of Human Behavior (2nd Ed.), Oxford : Elsevier, 130-136

Hofstadter, D. (2001). Analogy as the core of cognition ». In Gentner, D., Holyoak, K. J., Kokinov, B. N., The Analogical Mind : Perpectives from Cognitive Science. M.I.T. Press, 116-144.

Hofstadter, D. et Sander, E. (2013) L'Analogie, cour de la pensée. Paris : Odile Jacob.

Johsua, I. (2010). Quand 2009 questionne 1929. L'Économie politique, 48,(4), 64-84.

Kokinov, B., French, R. (2004). Humans - The Analogy Making Species, European Commission Sixth Framework: NEST-2004-Path-HUM. http://leadserv.u-bourgogne.fr/en/members/robertfrench/pages/humans-the-analogy-making-species [consulté le 23 mars 2018]

Ladmiral, J.-R. (2016). Analogie et littéralité à la lumière de la traduction. In Pamies, A. Monneret, Ph., Mejri, S (dir.), Analogie, figement et polysémie, Language Design, Special Issue.

Launay, M. (2004) Psychologie cognitive, Paris : Hachette. 
Leroy-Collombel, M. (2010) Eveil de la conscience grammaticale chez un enfant français entre 18 mois et 3 ans. In Neveu F., Muni Toke V., Durand J., Klingler T., Mondada L., Prévost S. (éds.) Congrès Mondial de Linguistique Française - CMLF 2010, 978-2-7598-0534-1, Paris, 2010, Institut de Linguistique Française, 1539-1555.

Monneret, Ph. (2004). Essais de linguistique analogique. Dijon : ABELL

Monneret, Ph. (2014). L'iconicité comme problème analogique. Le Français Moderne, 1. 46-77.

Monneret, Philippe. (2017a). Dénomination et analogie. In G. Petit, P. Haillet, X-L. Salvador, La dénomination : lexique et discours. Paris : Honoré Champion, 95-114.

Monneret, Ph. \& Albano, M. (2017b). De la composition comme dispositif analogique. Verbis, Lingue Letterature Culture anno VII, n. 2.

Papen, R., Bigot, D. (2010) Sontaient, ontvaient et fontsaient en français mitchif: variation et systématicité. In C. Leblanc, F. Martineau et Y. Frenette (dir.) Vues sur les français d'ici. Presses de l'Université Laval, 201-226.

Perelman, Ch. et Olbrechts-Tyteca, L. (1958). La nouvelle rhétorique. Traité de l'argumentation. Paris : Presses Universitaires de France.

Plantin, C. (2011). Analogie et métaphore argumentatives. A contrario, 16,(2), 110-130. https://www.cairn.info/revue-a-contrario-2011-2-page-110.htm. [consulté le 23 mars 2018]

Plantin, C. (2016). Dictionnaire de l'argumentation. Une introduction aux études d'argumentation Lyon : ENS Éditions.

Pottier, B. (1987) Théorie en analyse en linguistique. Paris : Hachette.

Saussure De, F. (1916) [1995]. Cours de linguistique générale, Paris : Payot.

Singer, I. B. (2004). Collected stories 1. Gimpel the Fool to The Letter Writer. New York : Library of America

Toulmin, S. E. (1958). The Uses of Argument. Cambridge: Cambridge University Press.

Toulmin, S. E., Rieke, R. \& Janik, A. (1984) An introduction to reasoning. New York : McMillan.

Truppa V, Piano Mortari E, Garofoli D, Privitera S, Visalberghi E (2011). Same/Different Concept Learning by Capuchin Monkeys in Matching-to-Sample, Tasks. PLoS ONE 6(8): e23809.

\footnotetext{
${ }^{1}$ L'identification est un processus scalaire. La similarité se décrit également sous la forme d'un continuum, entre les deux limites externes de l'identité et de la différence.

${ }^{2}$ «In the law, the principle of stare decisis effectively obliges the courts to reason from analogy. The principle directs judges to avoid unnecessary changes in legal practice by deciding similar cases in similar ways. Suppose that a case before the Court has the following essential facts:

1. A 27-year-old woman was

2. arrested for possession of a controlled substance and
} 
3 . confessed, but

4. she was not informed of her right to counsel or to silence

Lawyers for both the state and the defendant will then look for previously decided cases that share the same essential facts.

For instance, counsel for the defense will search out previous cases in which the one critical fact was the failure to advise the accused of the right to counsel or to silence. Reasoning from analogy, the court may then conclude that if previous cases were dismissed on this ground, the present case should also be dismissed. Counsel for the state will similarly look either for critical differences between the facts of the present and previous cases capable of destroying the analogy or for other precedent cases similar to the present case that were decided differently. » (Toulmin et al., 1984, 216-217)

3 Plantin précise que «le mécanisme de la catégorisation constitue la première étape d'une argumentation par la définition» (Plantin 2016, 122). Mais les exemples qu'il donne pour illustrer l'argumentation par la définition pourraient aussi bien être compris comme des cas d'argumentation par analogie catégorielle: "“tu es une fille, ma fille!": le savoir commun dit que les filles sont comme ça, doivent faire cela, etc. Donc ma fille, tu es comme ça et tu dois faire ainsi » (ibid., p. 193). L'inférence est présentée comme issue de la définition (ici d'une définition commune de « fille»), mais elle pourrait aussi être vue comme issue d'une opération de catégorisation («tu appartiens à la catégorie fille, donc...»).

${ }^{4}$ Pour un état des lieux récent sur la question de l'argumentation par métaphore, voir Bonhomme et al. (2017).

${ }^{5}$ «"L'imageabilité est une norme qui est, comme la familiarité conceptuelle, relative aux concepts. Il s'agit de la facilité, là encore estimée à partir d'échelles, avec laquelle un participant peut générer, à partir de la présentation visuelle du nom de l'image, une image mentale correspondant à l'objet (ou bien l'action s'il s'agit d'actions) désigné par le mot. Par exemple, à partir de la présentation visuelle du mot «pomme», l'individu doit s'en faire une représentation mentale - une image mentale - et il doit, à l'aide d'une échelle en x points, sélectionner la case qui correspond à son degré de facilité versus de difficulté d'élaboration de ladite image mentale. La case 1, par exemple, correspond à la réponse «je n'arrive pas à former une image mentale» ou «très difficilement» et la case 5 , au contraire, à la réponse «j'ai réalisé très facilement une image mentale». Pour le mot «pomme» par exemple, les valeurs d'imageabilité sont en général élevées » (Bonin 2016, 66). 